\title{
Kalidas Das
}

\section{Slip effects on heat transfer and peristaltic pumping of a Johnson-Segalman fluid in an inclined asymmetric channel}

Received: 16 March 2011 / Accepted: 22 November 2011 / Published online: 5 April 2012

(C) The Author(s) 2012. This article is published with open access at Springerlink.com

\begin{abstract}
This paper looks at the influence of slip conditions on heat transfer and the peristaltic flow of a Johnson-Segalman fluid in an inclined asymmetric channel under the supposition of long wave length. The asymmetry is produced by choosing the peristaltic wave train on the walls to have different amplitudes and phase. Both analytical and numerical solutions are presented. The analysis for the analytical solution is carried out for small Weissenberg number. The closed-form solutions have been obtained for the stream function, axial velocity, longitudinal pressure gradient and temperature. Numerical calculations are carried out for the pressure rise. The features of the flow and heat transfer characteristics are analysed by plotting graphs for different values of emerging parameters and discussed in detail.
\end{abstract}

Mathematics Subject Classification (2010) 76A05 · 76Z05

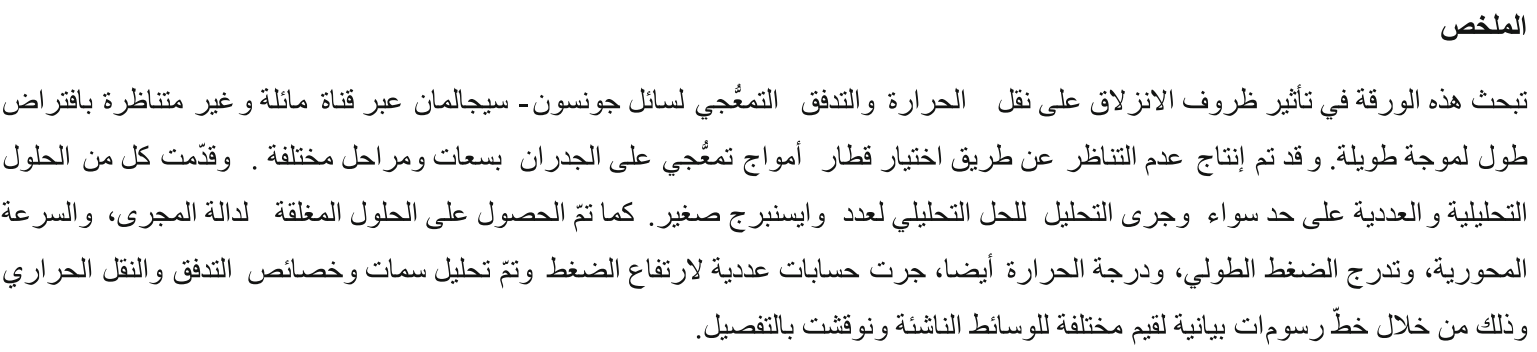

\section{Introduction}

The term peristalsis is used for the mechanism by which a fluid can be transported through a distensible tube when progressive waves of area contraction and expansion propagate along its length. In physiology, peristalsis is an important mechanism for transport of fluid and is used by the body to propel or mix the contents of a tube as in ureter, gastrointestinal tract, bile duct and other glandular ducts. Some biomedical instruments, like the blood pumps in dialysis and the heart lung machine use this principle. The mechanism of peristaltic transport has been exploited for industrial applications like sanitary fluid transport, transport of corrosive fluids where the contact of the fluid with the machinery parts is prohibited and transport of a toxic liquid used in

K. Das $(\varangle)$

Department of Mathematics,

Kalyani Government Engineering College, Kalyani,

Nadia, West Bengal 741235, India

E-mail:kd_kgec@rediffmail.com 
nuclear industry to avoid contamination of the outside environment. Extensive literature on the topic is available for viscous fluids. But the theory of non-Newtonian fluids has received great attention during the recent years, because the traditional viscous fluids cannot precisely describe characteristics of many physiological fluids. The governing equations for such fluids are complicated and highly non-linear and present interesting challenges to physicists, computer scientists, mathematicians and modellers.

The problem of the mechanism of peristaltic transport has attracted the attention of many investigators since the first investigation of Latham [17]. The fundamental studies on peristalsis were performed by Fung and Yih [6] using laboratory frame of reference and then by Shapiro et al. [32] using wave frame of reference. A number of analytical, numerical and experimental [1,2,7,9,20-26,31,34] studies have been conducted to understand peristaltic action for different kinds of fluids under different conditions with reference to physiological and mechanical situations. However, the interaction of peristalsis and heat transfer has not received much attention which may become highly relevant and significant in several industrial processes.Also thermodynamical aspects of blood may become significant in processes like oxygenation and hemodialysis when blood is drawn out of the body.Although there are many models to describe non-Newtonian behaviour of the fluids, the Johnson-Segalman fluid has acquired a special status in recent years, as it includes as special cases of classical Newtonian fluid, Oldroyd-B fluid and Maxwell fluid. The Johnson-Segalman model is a viscoelastic fluid model which was developed to allow for non-affine deformation [14]. Some researchers $[15,18,19]$ used this model to explain the phenomenon of 'spurt'. Peristaltic motion of Johnson-Segalman fluids in a planar channel was investigated by Hayat et al. [10]. Elshahed et al. [5] developed the model by considering effect of magnetic field. Literature survey bears witness to the fact that the information on peristalsis of non-Newtonian fluids in an asymmetric channel is scant. To the best of our knowledge Haroun [8] have studied the non-linear peristaltic motion of a fourth-grade fluid in an inclined asymmetric channel. Kothandapani and Srinivas [16] have investigated the influence of magnetic field on peristaltic transport of a Jeffrey fluid in an asymmetric channel. This model was further developed by Nadeem and Akram [27] by considering the effect of induced magnetic field. Srinivas and Pushparaj [33] considered non-linear peristaltic transport in an inclined asymmetric channel. Das [3] has investigated the effect of peristaltic pumping of a Johnson-Segalman fluid in an inclined asymmetric channel in the presence of external magnetic field. Recently, Nadeem and Akbar [28] developed the problem by considering heat and mass transfer effect on peristalsis flow of a Johnson-Segalman fluid in a vertical asymmetric channel with induced magnetic field.

It is well known that no-slip condition in polymeric liquids with high molecular weight is not appropriate. This condition also fails in many problems like thin film problem, rarefied fluid problem and flow on multiple interfaces. Again, when the molecular mean free path length of the fluid is comparable to the distance between the plates as in nanochannels or microchannels, the fluid exhibits non-continuum effects such as slip flow as demonstrated experimentally by Derek et al. [4]. Nadeem and Akram [29] have studied the problem of heat transfer in a peristaltic flow of MHD fluid with partial slip. Hayat et al. [12] have investigated the simultaneous effects of slip and heat transfer on the peristaltic flow in an asymmetric channel. Hayat and Mehmood [13] have discussed slip effects on MHD flow of third-order fluid in a planar channel. Recently, Nadeem and Akbar [30] examined the influence of heat transfer on peristaltic transport of a Johnson-Segalman fluid in an inclined asymmetric channel which has not been discussed so far when no-slip condition is no longer valid.

Therefore, the main purpose of the present study is to highlight the importance of slip conditions on heat transfer and peristaltic flow of Johnson-Segalman fluid in a two dimensional asymmetric inclined channel. The governing equations have been simplified using longwave length approximations. A perturbation solution is obtained for the case in which Weissenberg number is small. Numerical results are reported for various values of the physical parameters of interest. The paper has been arranged as follows: Section 2 deals with the formulation of the problems. In Sect. 3 we discuss the rate of volume flow and boundary conditions. Section 4 contains the closed form solutions of stream function, axial velocity, pressure gradient, pressure rise and temperature. Numerical results and discussion are presented in Sect. 5. The conclusions have been summarized in Sect. 6.

\section{Mathematical formulation of the problem}

Consider a two-dimensional infinite asymmetric inclined channel of width $d_{1}+d_{2}$ filled with an incompressible Johnson-Segalman fluid (see Fig. 1). We employ a rectangular co-ordinate system with $x$-axis parallel and $y$-axis normal to the channel. The channel flow is produced due to different amplitude and phases of the peristaltic waves with constant speed $c$ along the channel walls. The shape of the asymmetric channel walls are 


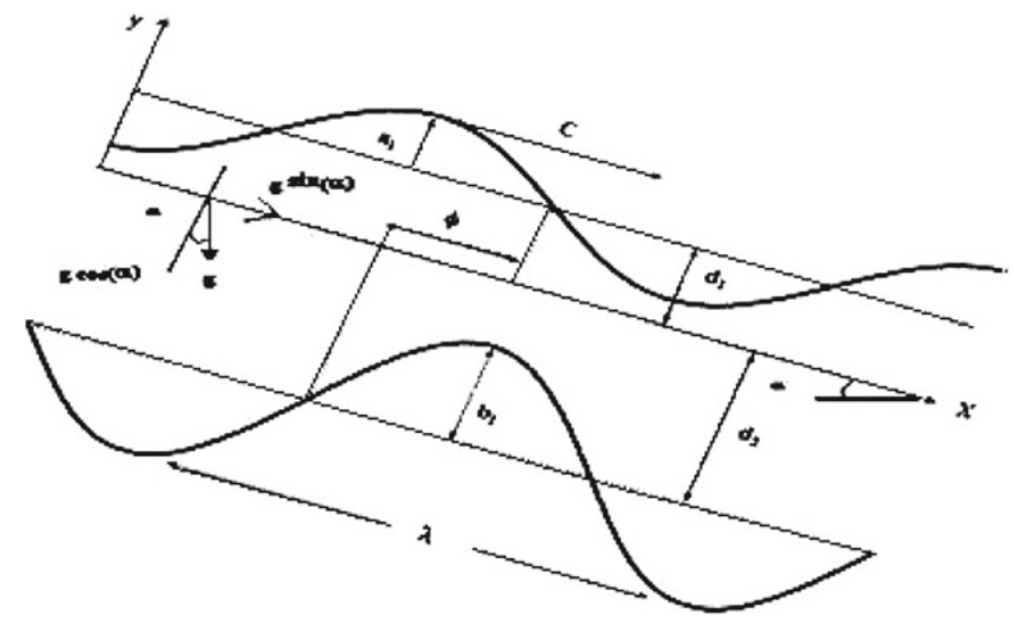

Fig. 1 Schematic diagram of the physical model

$$
\left.\begin{array}{l}
h_{1}=d_{1}+a_{1} \sin \frac{2 \pi}{\lambda}(X-c t), \text { upper wall } \\
h_{2}=-d_{2}-a_{2} \sin \left[\frac{2 \pi}{\lambda}(X-c t)+\phi\right], \text { lower wall }
\end{array}\right\}
$$

where $a_{1}$ and $a_{2}$ are the amplitudes of the waves, $\lambda$ is the wave length, $t$ is the time and $X$ is the direction of wave propagation. The phase difference $\phi$ varies in the range $0 \leq \phi \leq \pi$, in which $\phi=0$ corresponds to symmetric channel with waves out of phase and $\phi=\pi$ the waves are in phase and $a_{1}, b_{1}, d_{1}, d_{2}$ and $\phi$ satisfies the condition [11]

$$
a_{1}^{2}+a_{2}^{2}+2 a_{1} a_{2} \cos \phi \leq\left(d_{1}+d_{2}\right)^{2}
$$

The lower wall of the channel is maintained at temperature $T_{1}$ while the upper wall has temperature $T_{0}$

The equations governing the motion for the present problem are $[3,25,30]$

$$
\begin{gathered}
\frac{\partial U}{\partial X}+\frac{\partial V}{\partial Y}=0 \\
\rho\left(\frac{\partial}{\partial t}+U \frac{\partial}{\partial X}+V \frac{\partial}{\partial Y}\right) U=-\frac{\partial P}{\partial X}+\mu\left(\frac{\partial^{2}}{\partial X^{2}}+\frac{\partial^{2}}{\partial Y^{2}}\right) U+\frac{\partial S_{X X}}{\partial X}+\frac{\partial S_{X Y}}{\partial Y}+\rho g \sin \alpha \\
\rho\left(\frac{\partial}{\partial t}+U \frac{\partial}{\partial X}+V \frac{\partial}{\partial Y}\right) V=-\frac{\partial P}{\partial Y}+\mu\left(\frac{\partial^{2}}{\partial X^{2}}+\frac{\partial^{2}}{\partial Y^{2}}\right) V+\frac{\partial S_{X Y}}{\partial X}+\frac{\partial S_{Y Y}}{\partial Y}-\rho g \cos \alpha \\
2 \eta \frac{\partial U}{\partial X}=S_{X X}+m\left(\frac{\partial}{\partial t}+U \frac{\partial}{\partial X}+V \frac{\partial}{\partial Y}\right) S_{X X}-2 n m S_{X X} \frac{\partial U}{\partial X}+m\left[(1-n) \frac{\partial V}{\partial X}-(1+n) \frac{\partial U}{\partial Y}\right] S_{X Y} \\
\eta\left(\frac{\partial U}{\partial Y}+\frac{\partial V}{\partial X}\right)=S_{X Y}+m\left(\frac{\partial}{\partial t}+U \frac{\partial}{\partial X}+V \frac{\partial}{\partial Y}\right) S_{X Y}+\frac{m}{2}\left[(1-n) \frac{\partial U}{\partial Y}-(1+n) \frac{\partial V}{\partial X}\right] S_{X X} \\
+\frac{m}{2}\left[(1-n) \frac{\partial V}{\partial X}-(1+n) \frac{\partial U}{\partial Y}\right] S_{Y Y}, \\
2 \eta \frac{\partial V}{\partial Y}=S_{Y Y}+m\left(\frac{\partial}{\partial t}+U \frac{\partial}{\partial X}+V \frac{\partial}{\partial Y}\right) S_{Y Y}-2 n m S_{Y Y} \frac{\partial V}{\partial Y}+m\left[(1-n) \frac{\partial U}{\partial Y}-(1+n) \frac{\partial V}{\partial X}\right] S_{X Y}
\end{gathered}
$$




$$
\rho c_{\mathrm{p}}\left(\frac{\partial T}{\partial t}+U \frac{\partial T}{\partial X}+V \frac{\partial T}{\partial Y}\right)=S_{X X} \frac{\partial U}{\partial X}+S_{Y Y} \frac{\partial V}{\partial Y}+S_{X Y}\left(\frac{\partial V}{\partial X}+\frac{\partial U}{\partial Y}\right)+\kappa\left(\frac{\partial^{2} T}{\partial X^{2}}+\frac{\partial^{2} T}{\partial Y^{2}}\right)
$$

where $U, V$ are the velocities in the $X$ and $Y$ directions in the fixed frame, $\alpha$ is inclination of the channel with the horizontal, $P$ is the pressure, $\rho$ is the density, $\mu$ is the coefficient of viscosity of fluid, $g$ is the acceleration due to gravity, $T$ is the temperature, $c_{\mathrm{p}}$ is the specific heat at constant pressure and $\kappa$ is the thermal conductivity.

Let us introduce a wave frame $(x, y)$ moving with velocity c away from the fixed frame $(X, Y)$ by the transformation

$$
x=X-c t, y=Y, u=U-c, v=V, p(x)=P(X, t)
$$

where $u, v$ are the velocities in the $x$ and $y$ directions in the wave frame and $p$ is the pressure in wave frame.

Introducing the following non-dimensional quantities

$$
\begin{aligned}
& \bar{x}=\frac{x}{\lambda} ; \bar{y}=\frac{y}{d_{1}} ; \bar{u}=\frac{u}{c} ; \bar{v}=\frac{v}{c \delta} ; \delta=\frac{d_{1}}{\lambda} ; \bar{p}=\frac{d_{1}^{2} p}{c \lambda(\mu+\eta)} ; \bar{h}_{1}=\frac{h_{1}}{d_{1}} ; \bar{h}_{2}=\frac{h_{2}}{d_{1}} ; d=\frac{d_{2}}{d_{1}} ; \\
& \left.\begin{array}{l}
a=\frac{a_{1}}{d_{1}} ; b=\frac{a_{2}}{d_{1}} ; R=\frac{c d_{1} \rho}{\mu} ; R_{e}=\frac{c n \rho}{\mu} ; \bar{t}=\frac{c t}{\lambda} ; E_{c}=\frac{n^{2}}{c_{\mathrm{p}}\left(T_{0}-T_{1}\right)} ; F_{r}=\frac{c^{2}}{g d_{1}} ; W_{e}=\frac{m c}{d_{1}} ; \\
\bar{S}=\frac{d_{1} S}{\mu c} ; P_{r}=\frac{\rho c_{\mathrm{p}} v}{\kappa} ; \theta=\frac{T-T_{1}}{T_{0}-T_{1}}
\end{array}\right\}
\end{aligned}
$$

and the stream function $\psi$ so that $u=\frac{\partial \psi}{\partial y}, v=-\frac{\partial \psi}{\partial x}$ in (3)-(9), we finally get (after dropping bars)

$$
\begin{gathered}
\delta R_{e}\left(\frac{\partial \psi}{\partial y} \frac{\partial^{2} \psi}{\partial x \partial y}-\frac{\partial \psi}{\partial x} \frac{\partial^{2} \psi}{\partial y^{2}}\right)=-\frac{\mu+\eta}{\mu} \frac{\partial p}{\partial x}+\delta \frac{\partial S_{x x}}{\partial x}+\frac{\partial S_{x y}}{\partial y}+\left(\delta^{2} \frac{\partial^{3} \psi}{\partial^{2} x \partial y}+\frac{\partial^{3} \psi}{\partial y^{3}}\right)+\frac{R \sin \alpha}{F_{r}} \\
-\delta^{3} R_{e}\left(\frac{\partial \psi}{\partial y} \frac{\partial^{2} \psi}{\partial x^{2}}-\frac{\partial \psi}{\partial x} \frac{\partial^{2} \psi}{\partial x \partial y}\right)=-\frac{\mu+\eta}{\mu} \frac{\partial p}{\partial y}+\delta^{2} \frac{\partial S_{x y}}{\partial x}+\delta \frac{\partial S_{y y}}{\partial y}-\delta^{2}\left(\delta^{2} \frac{\partial^{3} \psi}{\partial x^{3}}+\frac{\partial^{3} \psi}{\partial x \partial^{2} y}\right)-\delta \frac{R \cos \alpha}{F_{r}} \\
\frac{2 \eta \delta}{\mu} \frac{\partial^{2} \psi}{\partial x \partial y}=S_{x x}+W_{e} \delta\left(\frac{\partial \psi}{\partial y} \frac{\partial S_{x x}}{\partial x}-\frac{\partial \psi}{\partial x} \frac{\partial S_{x x}}{\partial y}\right)-2 W_{e} n \delta S_{x x} \frac{\partial^{2} \psi}{\partial x \partial y}-W_{e}\left\{\delta^{2}(1-n) \frac{\partial^{2} \psi}{\partial x^{2}}+(1+n) \frac{\partial^{2} \psi}{\partial y^{2}}\right\} S_{x y} \\
\frac{\eta}{\mu}\left(\frac{\partial^{2} \psi}{\partial y^{2}}-\delta^{2} \frac{\partial^{2} \psi}{\partial x^{2}}\right)=S_{x y}+W_{e} \delta\left(\frac{\partial \psi}{\partial y} \frac{\partial S_{x y}}{\partial x}-\frac{\partial \psi}{\partial x} \frac{\partial S_{x y}}{\partial y}\right)+\frac{W_{e}}{2}\left\{\delta^{2}(1+n) \frac{\partial^{2} \psi}{\partial x^{2}}+(1-n) \frac{\partial^{2} \psi}{\partial y^{2}}\right\} S_{x x} \\
-\frac{W_{e}}{2}\left\{\delta^{2}(1-n) \frac{\partial^{2} \psi}{\partial x^{2}}+(1+n) \frac{\partial^{2} \psi}{\partial y^{2}}\right\} S_{y y} \\
-\frac{2 \eta \delta}{\mu} \frac{\partial^{2} \psi}{\partial x \partial y}=S_{y y}+W_{e} \delta\left(\frac{\partial \psi}{\partial y} \frac{\partial S_{y y}}{\partial x}-\frac{\partial \psi}{\partial x} \frac{\partial S_{y y}}{\partial y}\right)+2 W_{e} n \delta S_{y y} \frac{\partial^{2} \psi}{\partial x \partial y}+W_{e}\left\{\delta^{2}(1+n) \frac{\partial^{2} \psi}{\partial x^{2}}+(1-n) \frac{\partial^{2} \psi}{\partial y^{2}}\right\} S_{x y} \\
\delta R_{e}\left(\frac{\partial \psi}{\partial y} \frac{\partial \theta}{\partial x}-\delta \frac{\partial \psi}{\partial x} \frac{\partial \theta}{\partial y}\right)=E_{c}\left(S_{x x} \delta \frac{\partial^{2} \psi}{\partial x \partial y}-S_{x y} \delta \frac{\partial^{2} \psi}{\partial x^{2}}+S_{y x} \frac{\partial^{2} \psi}{\partial y^{2}}-S_{y y} \delta \frac{\partial^{2} \psi}{\partial y \partial x}\right)+\frac{1}{P_{r}}\left(\delta^{2} \frac{\partial^{2} \theta}{\partial x^{2}}+\frac{\partial^{2} \theta}{\partial y^{2}}\right)
\end{gathered}
$$

where $R$ is the Reynolds number, $\delta$ is the wave number, $F_{r}$ is the Froude number, $W_{e}$ is the Weissenberg number, $E_{c}$ is the Eckert number and $P_{r}$ is the Prandtl number. 


\section{Rate of volume flow and boundary conditions}

The dimensional volume flow rate in the fixed frame is given by

$$
Q=\int_{h_{2}(X)}^{h_{1}(X)} U(X, Y, t) \mathrm{d} Y
$$

In the wave frame, Eq. (18) reduces to

$$
q=\int_{h_{2}(x)}^{h_{1}(x)} u(x, y) \mathrm{d} y
$$

Using Eq. (10) in Eqs. (18) and (19), we have

$$
Q=q+c h_{1}-c h_{2}
$$

The average volume flow rate over one period $\left(T=\frac{\lambda}{c}\right)$ of the peristaltic wave is defined as

$$
\bar{Q}=\frac{1}{T} \int_{0}^{T} Q \mathrm{~d} t
$$

Then using Eq. (20), we receive

$$
\bar{Q}=q+c d_{1}+c d_{2}
$$

Defining the dimensionless mean flows

$$
\Theta=\frac{\bar{Q}}{c d_{1}}, \quad F=\frac{q}{c d_{1}}
$$

in fixed and moving frames, respectively, we can write (23) as

$$
\Theta=F+1+d
$$

where

$$
F=\int_{h_{2}}^{h_{1}} \frac{\partial \psi}{\partial y} \mathrm{~d} y=\psi\left(h_{1}\right)-\psi\left(h_{2}\right)
$$

and the dimensionless peristaltic walls are

$$
\left.\begin{array}{l}
h_{1}=1+a \sin 2 \pi x \\
h_{2}=-d-b \sin (2 \pi x+\phi)
\end{array}\right\}
$$

where $a, b, \phi$ and $d$ satisfy the relation

$$
a^{2}+b^{2}+2 a b \cos \phi \leq(1+d)^{2}
$$

Since we are considering the slip flow on the wall, the dimensionless boundary conditions for the present problem in the wave frame can be written as $[12,29]$

$$
\left.\begin{array}{l}
\psi=\frac{F}{2}, \frac{\partial \psi}{\partial y}+\beta \frac{\partial^{2} \psi}{\partial y^{2}}=-1, \theta+\gamma \frac{\partial \theta}{\partial y}=1, \text { at } \quad y=h_{1}(x), \\
\psi=-\frac{F}{2}, \frac{\partial \psi}{\partial y}-\beta \frac{\partial^{2} \psi}{\partial y^{2}}=-1, \theta-\gamma \frac{\partial \theta}{\partial y}=0, \text { at } \quad y=h_{2}(x) .
\end{array}\right\}
$$

where $\beta$ is the non-dimensional slip velocity parameter (Knudsen number) and $\gamma$ is the non-dimensional thermal slip parameter. 


\section{Solution of the problem}

\subsection{Equations for large wavelength}

As the Eqs. (12)-(17) are highly non-linear differential equations, analytical solutions valid for all arbitrary parameters involved in these equations seem to be impossible to find. Therefore, we carry out our investigation on the basis that the dimensionless wave number $\delta$ is small, that is, $\delta \ll 1$, which corresponds to the long wavelength approximation. Then employing long wavelength approximation, one can find from (12)-(17) that

$$
\begin{gathered}
\frac{\mu+\eta}{\mu} \frac{\partial p}{\partial x}=\frac{\partial S_{x y}}{\partial y}+\frac{\partial^{3} \psi}{\partial y^{3}}+\frac{R \sin \alpha}{F_{r}}, \\
0=-\frac{\partial p}{\partial y}, \\
S_{x x}-W_{e}(1+n) S_{x y} \frac{\partial^{2} \psi}{\partial y^{2}}=0, \\
\frac{\eta}{\mu} \frac{\partial^{2} \psi}{\partial y^{2}}=S_{x y}+\frac{W_{e}}{2}(1-n) S_{x x} \frac{\partial^{2} \psi}{\partial y^{2}}-\frac{W_{e}}{2}(1+n) S_{y y} \frac{\partial^{2} \psi}{\partial y^{2}}, \\
S_{y y}+W_{e}(1-n) S_{x y} \frac{\partial^{2} \psi}{\partial y^{2}}=0, \\
E_{c} P_{r} S_{y x} \frac{\partial^{2} \psi}{\partial y^{2}}+\frac{\partial^{2} \theta}{\partial y^{2}}=0
\end{gathered}
$$

Equation (30) shows that $p$ is not a function of $y$. Then after rearrangement, we get from the above equations

$$
\begin{gathered}
\frac{\partial^{2}}{\partial y^{2}}\left[\frac{\left(\frac{\eta}{\mu}+1\right) \frac{\partial^{2} \psi}{\partial y^{2}}+W_{e}^{2}\left(1-n^{2}\right)\left(\frac{\partial^{2} \psi}{\partial y^{2}}\right)^{3}}{1+W_{e}^{2}\left(1-n^{2}\right)\left(\frac{\partial^{2} \psi}{\partial y^{2}}\right)^{2}}\right]=0 \\
\frac{\mu+\eta}{\mu} \frac{\mathrm{d} p}{\mathrm{~d} x}=\frac{\partial}{\partial y}\left[\frac{\frac{\eta}{\mu} \frac{\partial^{2} \psi}{\partial y^{2}}}{1+W_{e}^{2}\left(1-n^{2}\right)\left(\frac{\partial^{2} \psi}{\partial y^{2}}\right)^{2}}\right]+\frac{\partial^{3} \psi}{\partial y^{3}}+\frac{R \sin \alpha}{F_{r}}, \\
\frac{\partial^{2} \theta}{\partial y^{2}}=-E_{c} P_{r}\left[\frac{\eta}{\mu}\left(\frac{\partial^{2} \psi}{\partial y^{2}}\right)^{2}-W_{e}^{2} \frac{\eta}{\mu}\left(1-n^{2}\right)\left(\frac{\partial^{2} \psi}{\partial y^{2}}\right)^{4}\right]
\end{gathered}
$$

\subsection{Perturbation solution}

For small values of $W_{e}^{2}$, Eqs. (35), (36) can be written using the binomial theorems as

$$
\begin{gathered}
\frac{\partial^{2}}{\partial y^{2}}\left[\frac{\partial^{2} \psi}{\partial y^{2}}+W_{e}^{2} \alpha_{1}\left(\frac{\partial^{2} \psi}{\partial y^{2}}\right)^{3}+W_{e}^{4} \alpha_{2}\left(\frac{\partial^{2} \psi}{\partial y^{2}}\right)^{5}\right]=0, \\
\frac{\mathrm{d} p}{\mathrm{~d} x}=\frac{\partial^{3} \psi}{\partial y^{3}}+\frac{R \sin \alpha}{F_{r}}\left(\frac{\mu}{\mu+\eta}\right)+W_{e}^{2} \alpha_{1} \frac{\partial}{\partial y}\left(\frac{\partial^{2} \psi}{\partial y^{2}}\right)^{3}+W_{e}^{4} \alpha_{2} \frac{\partial}{\partial y}\left(\frac{\partial^{2} \psi}{\partial y^{2}}\right)^{5},
\end{gathered}
$$

where

$$
\alpha_{1}=\frac{\left(n^{2}-1\right) \eta}{\mu+\eta}, \quad \alpha_{2}=\left(n^{2}-1\right) \alpha_{1}
$$


Now, we seek the solutions of (37)-(39) with the boundary conditions (28) for a small Weissenberg number. We expand flow quantities in a power series of $W_{e}^{2}$ as follows:

$$
\begin{aligned}
& \psi=\psi_{0}+W_{e}^{2} \psi_{1}+0\left(W_{e}^{4}\right), \\
& p=p_{0}+W_{e}^{2} p_{1}+0\left(W_{e}^{4}\right), \\
& F=F_{0}+W_{e}^{2} F_{1}+0\left(W_{e}^{4}\right), \\
& \theta=\theta_{0}+W_{e}^{2} \theta_{1}+0\left(W_{e}^{4}\right)
\end{aligned}
$$

Substituting (41) into Eqs. (37-39) and then finding the solutions of all systems we arrive at the final solutions which are defined as

$$
\begin{aligned}
\psi= & C_{1} y\left\{6\left(h_{1} h_{2}-h_{1} \beta+h_{2} \beta\right)-3 y\left(h_{1}+h_{2}\right)+2 y^{2}\right\}-y+C_{2} \\
+ & W_{e}^{2}\left[\frac{1}{6} C_{4}\left\{y^{2}\left(3 h_{1}+3 h_{2}-2 y\right)-y\left(h_{1}^{2}+4 h_{1} h_{2}+h_{2}^{2}\right)+h_{1} h_{2}\left(h_{1}+h_{2}\right)\right\}\right. \\
- & \left.C_{5}\left(y-h_{1}\right)\left(y-h_{2}\right)\left(2 y-h_{1}-h_{2}\right)\left\{2 y^{2}-2 y\left(h_{1}+h_{2}\right)+h_{1}^{2}+h_{2}^{2}\right\}+\frac{F_{1}\left(2 y-h_{1}-h_{2}\right)}{2\left(h_{1}-h_{2}\right)}\right], \\
\theta= & C_{1}\left(h_{1}-h_{2}\right)+C_{3}\left(2 y-h_{1}-h_{2}\right)^{4}+\frac{y+\gamma-h_{2}}{h_{1}-h_{2}+2 \gamma} \\
& +W_{e}^{2}\left[-\frac{E_{c} P_{r} \eta}{1,680 \mu}\left[35 C_{4}\left(2 y-h_{1}-h_{2}\right)^{4}+224 C_{5}\left(2 y-h_{1}-h_{2}\right)^{6}\left\{C_{4}+81 C_{5}\left(n^{2}-1\right)\right\}\right.\right. \\
& \left.\left.+480 C_{5}^{2}\left(2 y-h_{1}-h_{2}\right)^{8}\right]+C_{6}\right] \\
& \frac{\mathrm{d} p}{\mathrm{~d} x}=12 C_{1}+\frac{R \mu \sin \alpha}{F_{r}(\mu+\eta)}+W_{e}^{2}\left[\frac{4\left\{C_{5}\left(h_{1}-h_{2}\right)^{5}-F_{1}\right\}}{\left(h_{1}-h_{2}\right)^{2}\left\{\left(h_{1}-h_{2}\right)^{2}+6 \beta\right\}}\right]
\end{aligned}
$$

Then the axial velocity $u$ is given by

$$
\begin{aligned}
u= & 6 C_{1}\left\{\left(h_{1} h_{2}-h_{1} \beta+h_{2} \beta\right)-y\left(h_{1}+h_{2}\right)+y^{2}\right\}-1 \\
& +W_{e}^{2}\left[\frac{1}{6} C_{4}\left\{6 y\left(h_{1}+h_{2}-y\right)-\left(h_{1}^{2}+4 h_{1} h_{2}+h_{2}^{2}\right)\right\}\right. \\
& \left.+\frac{1}{8} C_{5}\left\{5\left(2 y-h_{1}-h_{2}\right)^{4}-\left(h_{1}-h_{2}\right)^{4}\right\}+\frac{F_{1}}{h_{1}-h_{2}}\right],
\end{aligned}
$$

where

$$
\begin{gathered}
C_{1}=-\frac{F_{0}+h_{1}-h_{2}}{\left(h_{1}-h_{2}\right)^{2}\left(6 \beta+h_{1}-h_{2}\right)}, \\
C_{2}=\frac{F_{0}}{2}+h_{1}-h_{1} C_{1}\left(3 h_{1} h_{2}-6 h_{1} \beta+6 h_{2} \beta-h_{1}^{2}\right), \\
C_{3}=-\frac{3 E_{c} P_{r} \eta C_{1}^{2}}{4 \mu}, \\
C_{4}=\frac{2 F_{1}-C_{5}\left(h_{1}-h_{2}\right)^{5}}{\left(h_{1}-h_{2}\right)^{2}\left\{\left(h_{1}-h_{2}\right)^{2}+6 \beta\right\}} \\
C_{5}=\frac{108 C_{2}^{3} \mu\left(n^{2}-1\right)}{5(\mu+\eta)}
\end{gathered}
$$


and

$$
\begin{aligned}
C_{6}= & \frac{E_{c} P_{r} \eta\left(h_{1}-h_{2}\right)^{3}}{1,680 \mu}\left[35 C_{4}^{2}\left(8 \gamma+h_{1}-h_{2}\right)+224 C_{5}\left(h_{1}-h_{2}\right)^{2}\left(12 \gamma+h_{1}-h_{2}\right)\left\{C_{4}+81 C_{5}\left(n^{2}-1\right)\right\}\right. \\
& \left.+480 C_{5}^{2}\left(h_{1}-h_{2}\right)^{4}\left(16 \gamma+h_{1}-h_{2}\right)\right]
\end{aligned}
$$

The non-dimensional expression for pressure rise per wavelength $\Delta P_{\lambda}$ is given by

$$
\Delta P_{\lambda}=\int_{0}^{1} \frac{\mathrm{d} p}{\mathrm{~d} x} \mathrm{~d} x
$$

where $\frac{\mathrm{d} p}{\mathrm{~d} x}$ is defined through Eq. (44).

Note that if we put the slip parameters $\beta$ and $\gamma$ equal to zero, then the results of the problem reduce exactly to the same as that found by Nadeem and Akbar [30].

\section{Numerical results and discussion}

This section aims to analyse the behaviour of the streamlines, axial velocity,pressure gradient pressure rise and temperature graphically for embedded flow parameters in the present problem. The expression for pressure rise is calculated numerically using MATHEMATICA.

An interesting phenomenon of peristaltic motion in the wave frame is trapping which is basically the formation of an internally circulating bolus of fluid by closed streamlines. This trapped bolus is pushed ahead with the peristaltic wave. This trapping phenomena for different values of $W_{e}, \beta$ and $\Theta$ are shown in Figs. 2, $3,4,5,6,7,8$. It is observed from Figs. 2 and 3 that the trapped bolus which are moving as whole decreases in size with the decrease in $\Theta$. The effects of Weissenberg number $W_{e}$ on trapping can be seen from Figs. 2, 4 , 5. It is depicted that increase in $W_{e}$ the trapping bolus which is moving as a whole increases.These results are in agreement with the results obtained by Nadeem and Akbar [30]. The effect of slip parameter $\beta$ on the trapping is illustrated in Figs. 6, 7 and 8, and it is observed that the size of trapped bolus rapidly decreases with increasing $\beta$.

To study the behaviour of the distributions of the axial velocity $u$, numerical calculations for several values of $\beta, W_{e}$ and $\phi$ are carried out in Figs. 9, 10 and 11. Figure 9 shows that an increase in $\beta$ results in decrease of velocity distribution near the wall but the effect is negligible at the middle portion of the channel. Figure 10 displays that with an increase in $W_{e}$, the velocity increases slightly but there is no effect at the centre of the channel.The influence of $\phi$ on velocity distribution is shown in Fig. 11, and it is observed that the axial

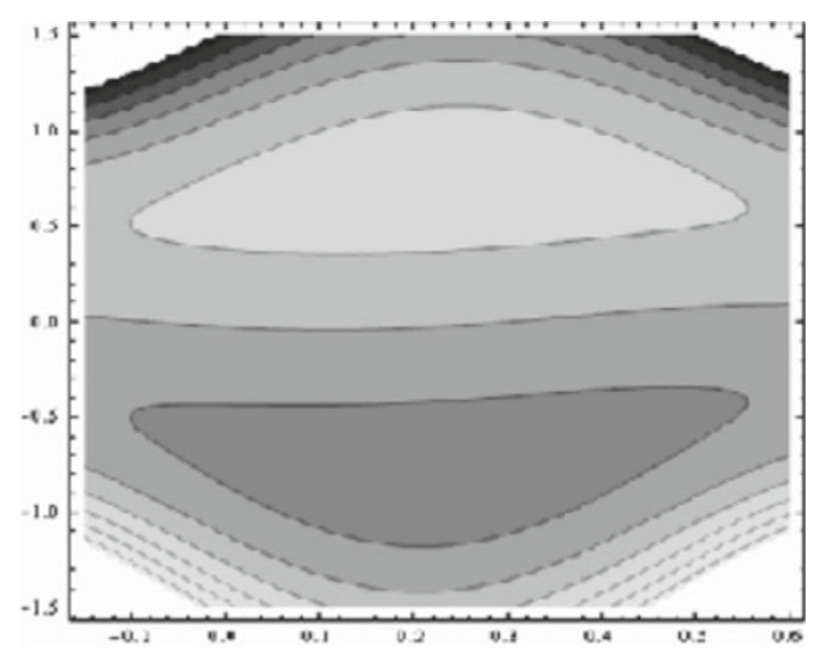

Fig. 2 Streamlines for different values of $W_{e}, \beta, \Theta: W_{e}=0.04, \beta=0.02, \Theta=1.93$ 


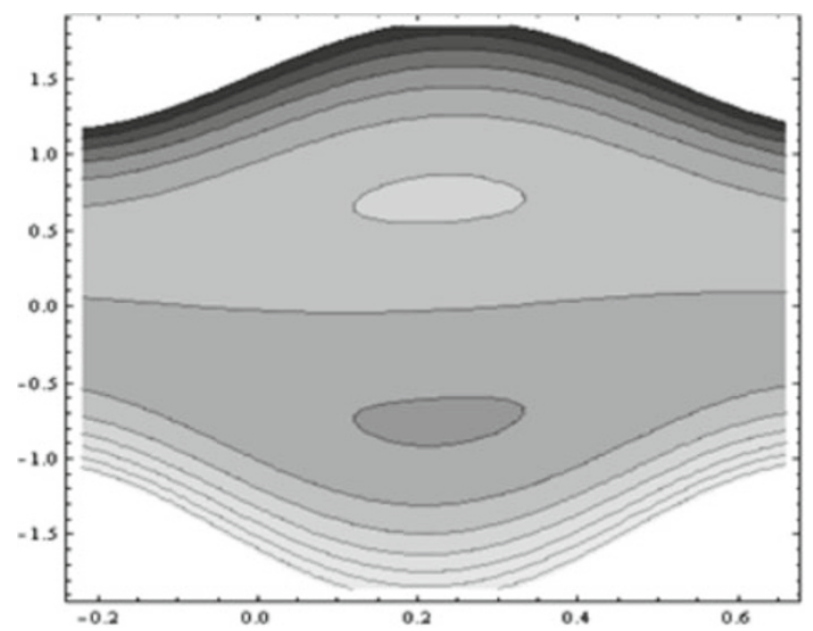

Fig. 3 Streamlines for different values of $W_{e}, \beta, \Theta: W_{e}=0.04, \beta=0.02, \Theta=1.87$

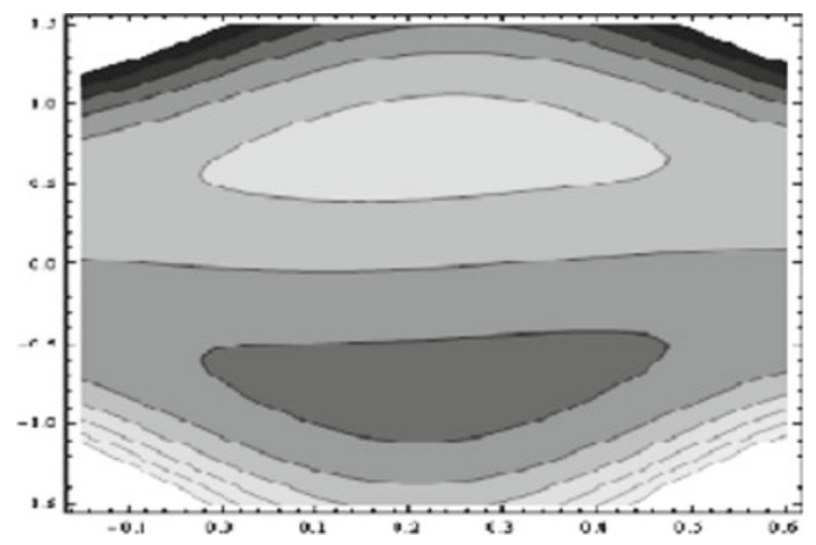

Fig. 4 Streamlines for different values of $W_{e}, \beta, \Theta: W_{e}=0.02, \beta=0.02, \Theta=1.93$

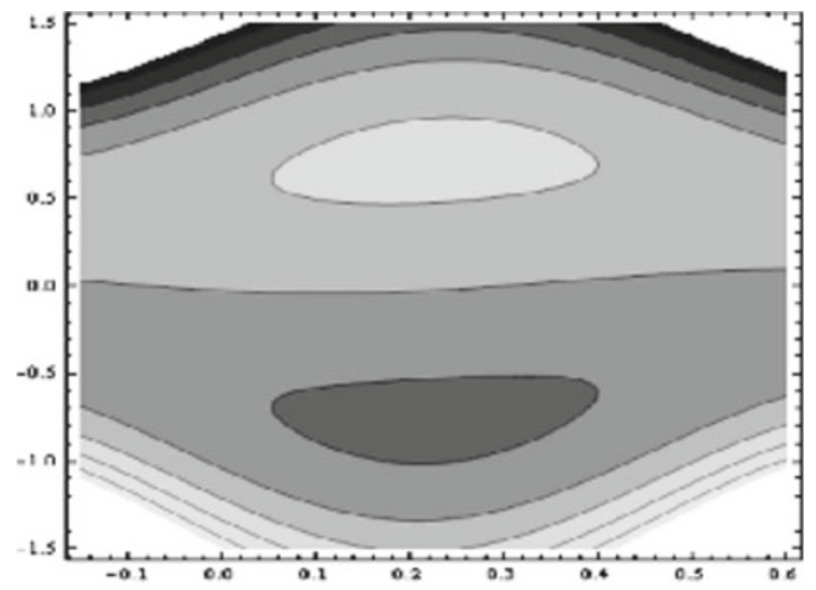

Fig. 5 Streamlines for different values of $W_{e}, \beta, \Theta: W_{e}=0.00, \beta=0.02, \Theta=1.93$

velocity increases with an increase of the phase angle $\phi$ but the effect is not prominent at the upper portion of the channel.

Figures 12, 13 and 14 are prepared for the pressure gradient against $x$ for different values of $\beta, F_{r}$, and $\alpha$ It can be noticed that in the wider part of the channel $x \varepsilon[0,0.1]$ and $[0.4,0.5]$, the pressure gradient is relatively small, that is, the flow can easily pass without imposition of a large pressure gradient. On the other hand,in a 


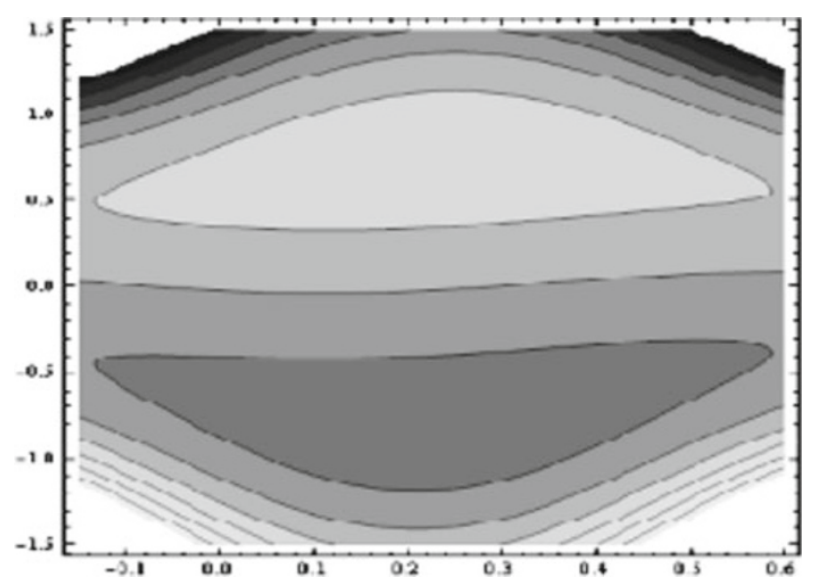

Fig. 6 Streamlines for different values of $W_{e}, \beta, \Theta: W_{e}=0.04, \beta=0.00, \Theta=1.93$

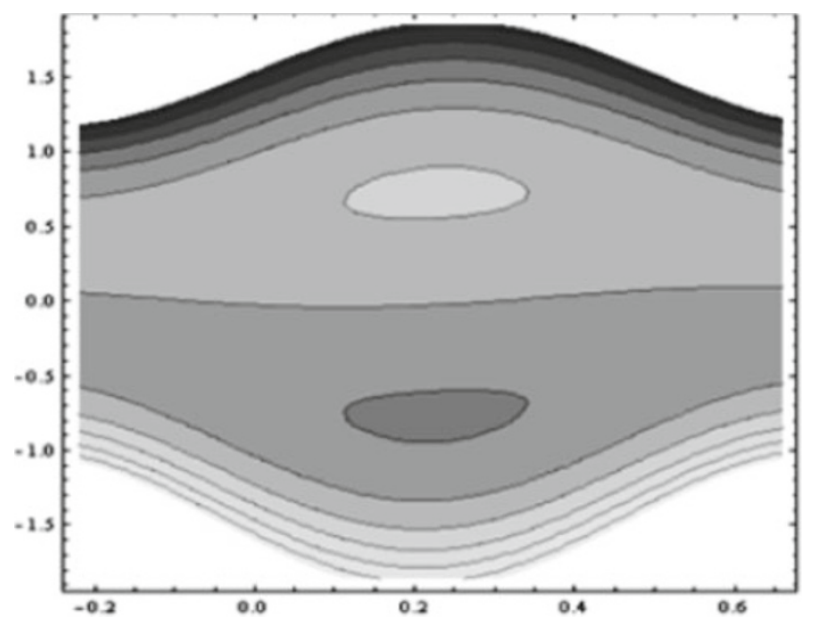

Fig. 7 Streamlines for different values of $W_{e}, \beta, \Theta: W_{e}=0.04, \beta=0.06, \Theta=1.93$

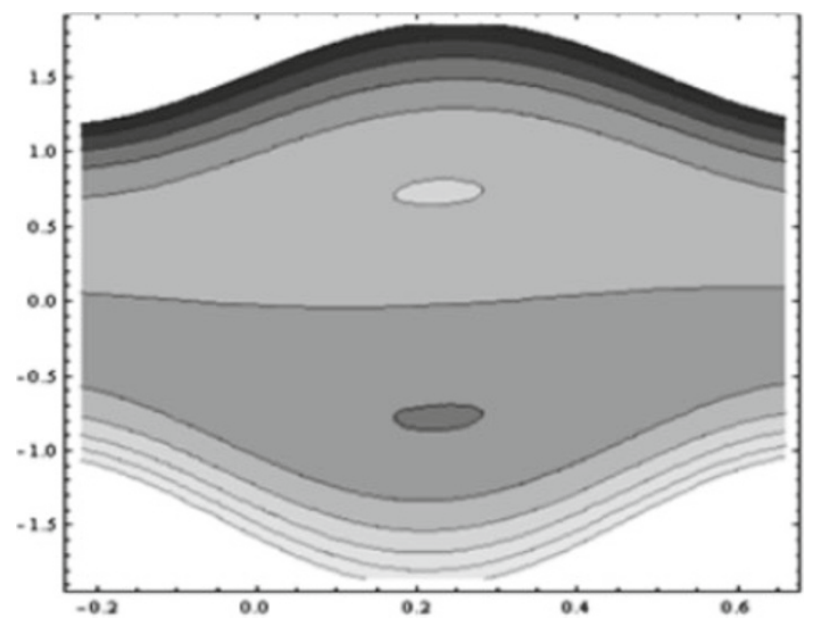

Fig. 8 Streamlines for different values of $W_{e}, \beta, \Theta: W_{e}=0.04, \beta=0.09, \Theta=1.93$ 


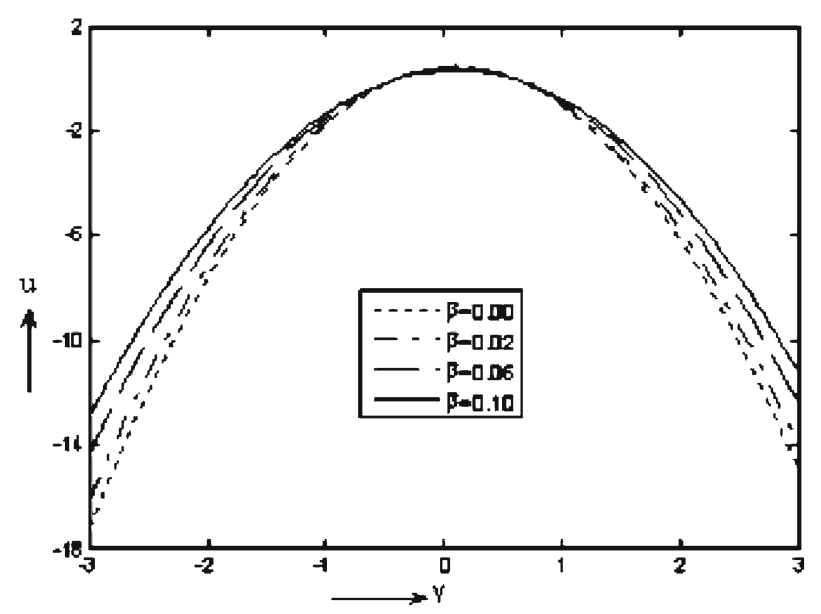

Fig. 9 Velocity distribution for different values of $\beta: a=0.25, b=0.4, d=0.95, x=0.5$

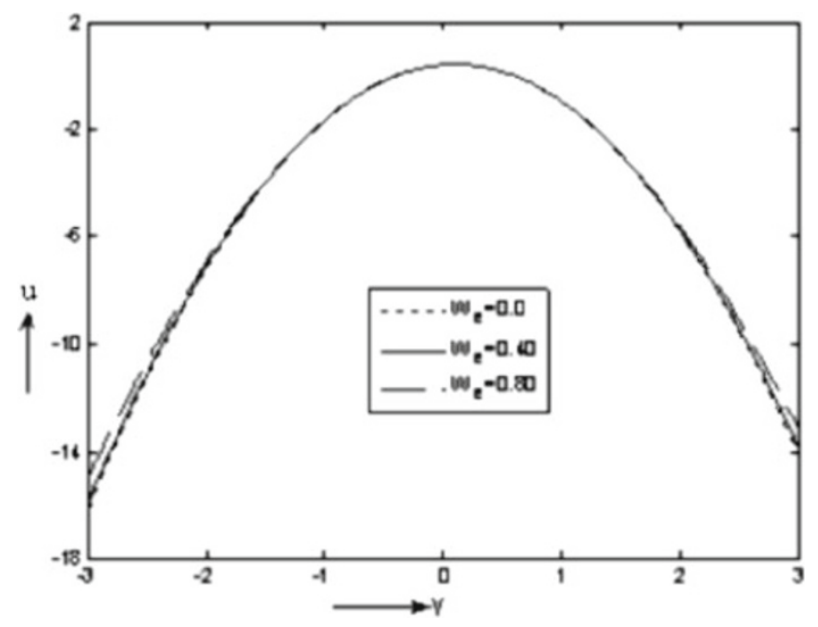

Fig. 10 Velocity distribution for different values of $W_{e}: a=0.25, b=0.4, d=0.95, x=0.5$

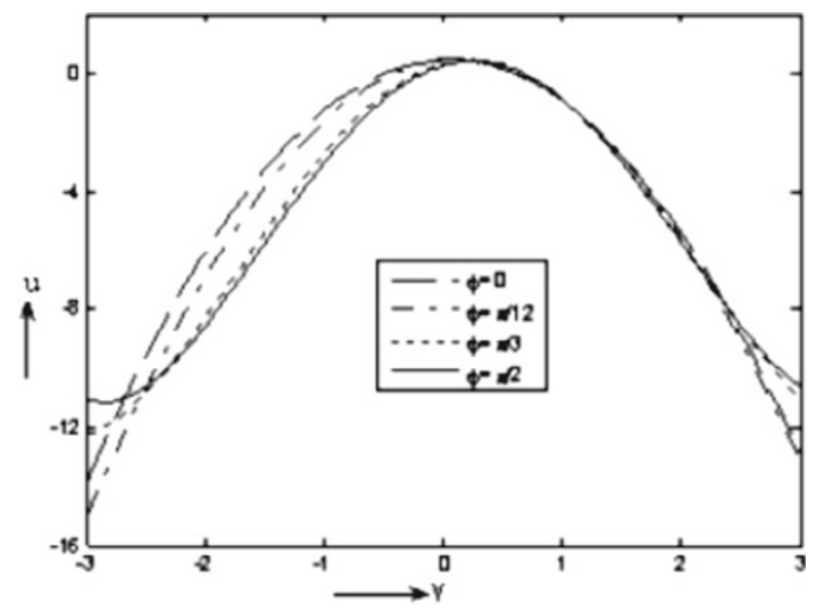

Fig. 11 Velocity distribution for different values of $\phi: a=0.25, b=0.4, d=0.95, x=0.5$ 


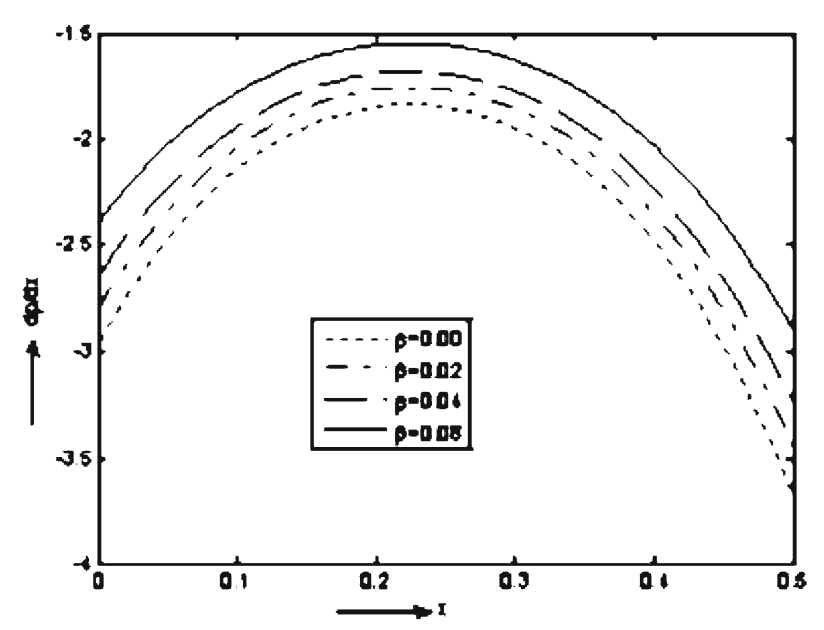

Fig. 12 Variation of the pressure gradient with $x$ for different values of $\beta: a=0.25, b=0.4, d=0.95$

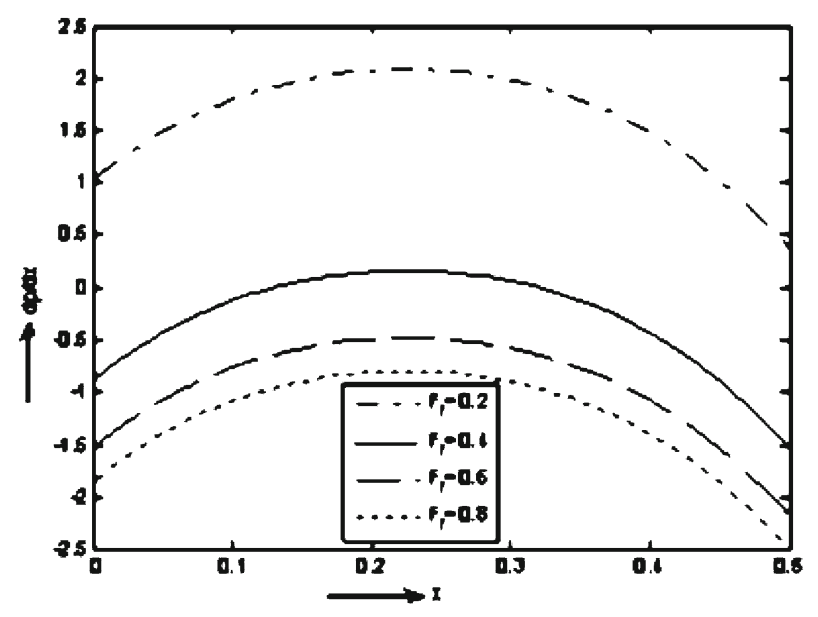

Fig. 13 Variation of the pressure gradient with $x$ for different values of $F_{r}: a=0.25, b=0.4, d=0.95$

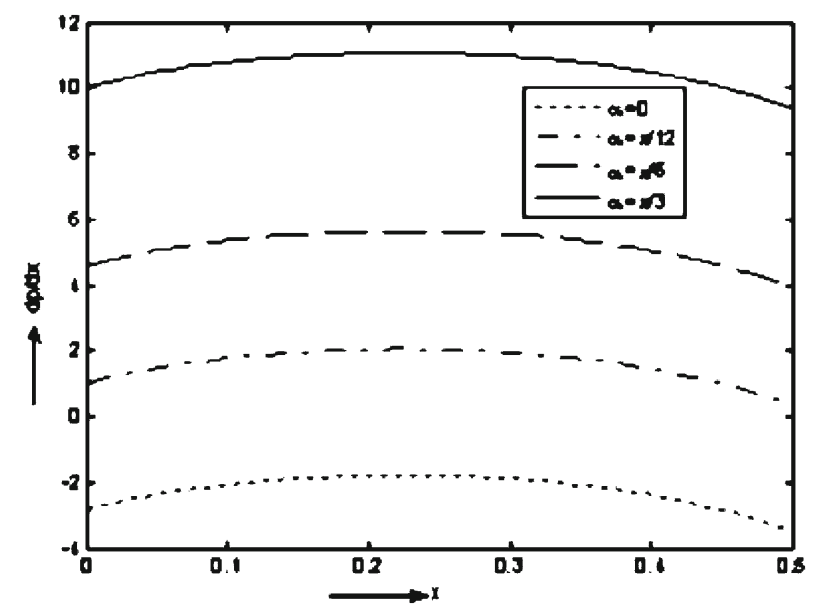

Fig. 14 Variation of the pressure gradient with $x$ for different values of $\alpha: a=0.25, b=0.4, d=0.9$ 


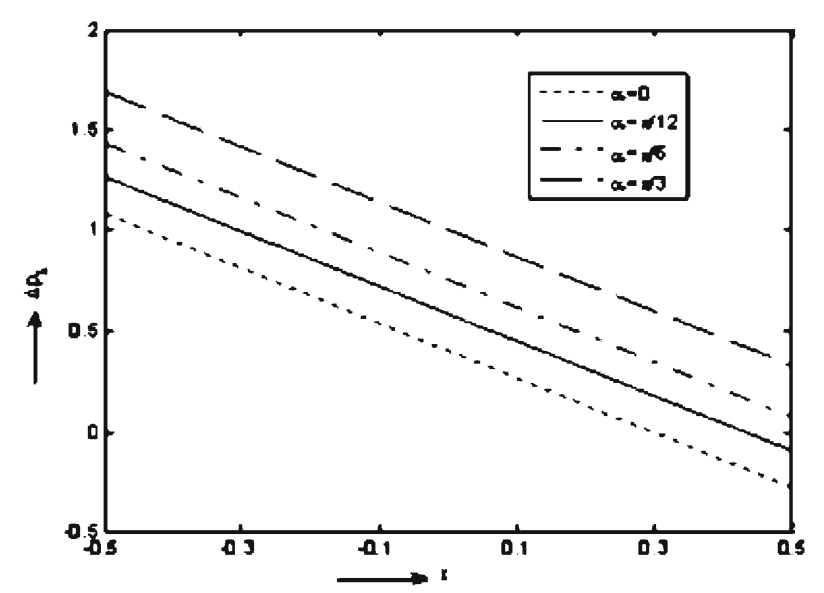

Fig. 15 Variation of the pressure rise with $x$ for different values of $\alpha: a=0.25, b=0.4, d=0.95$

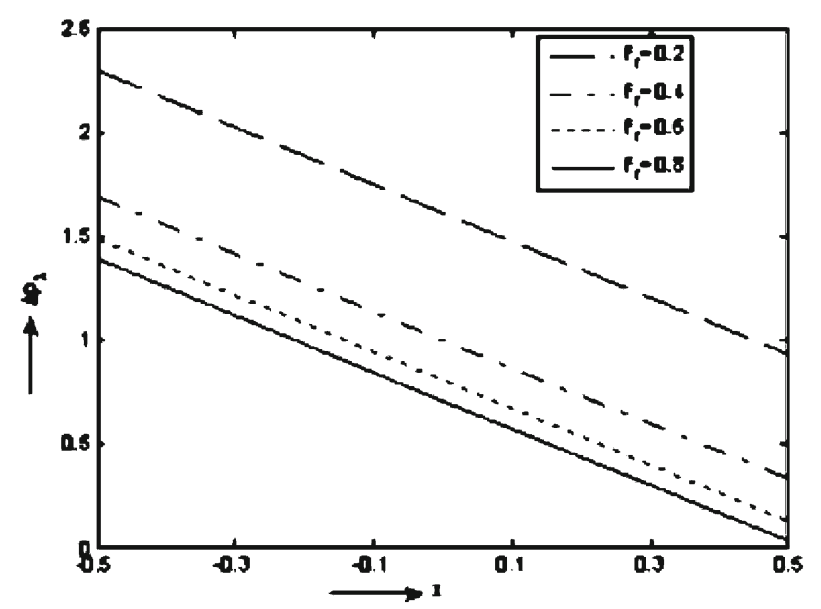

Fig. 16 Variation of the pressure rise with $x$ for different values of $F_{r}: a=0.25, b=0.4, d=0.95$

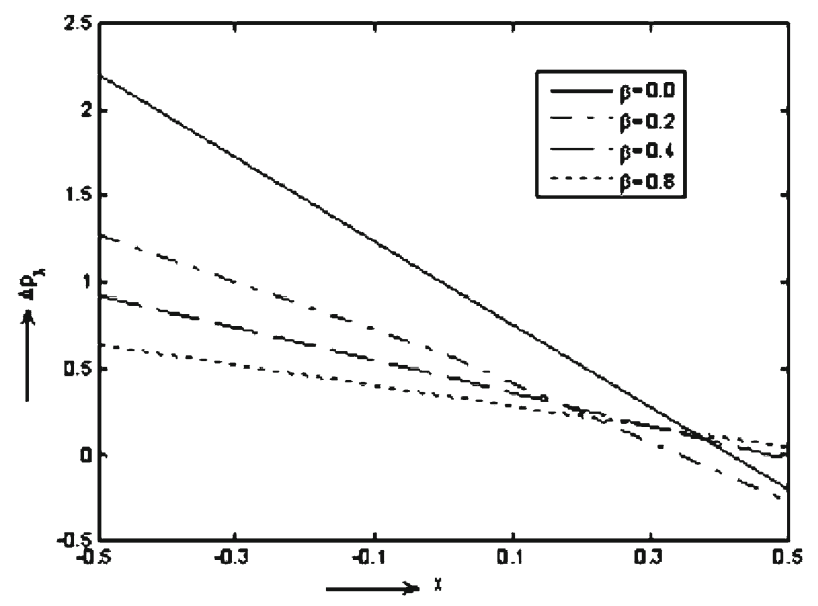

Fig. 17 Variation of the pressure rise with $x$ for different values of $\beta: a=0.25, b=0.4, d=0.95$ 


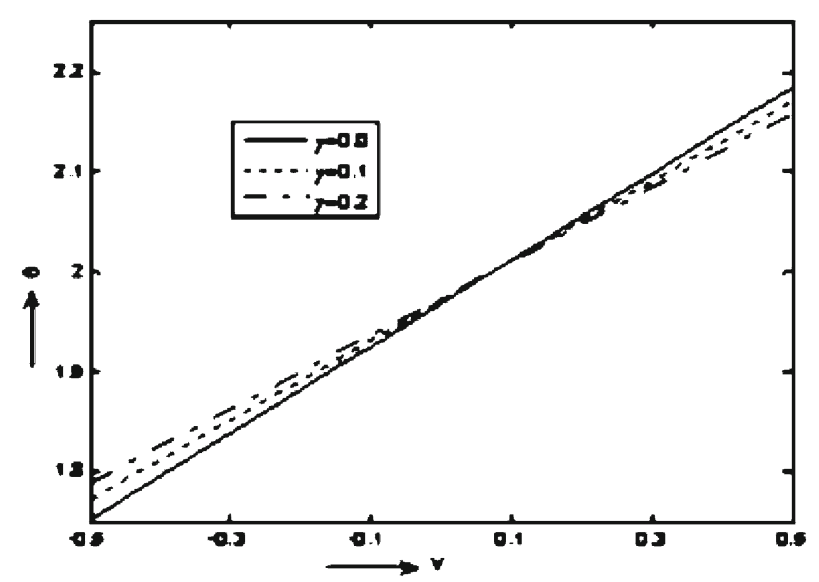

Fig. 18 Variation of temperature with y for different values of $\gamma: a=0.25, b=0.4, d=0.95, x=0.5$

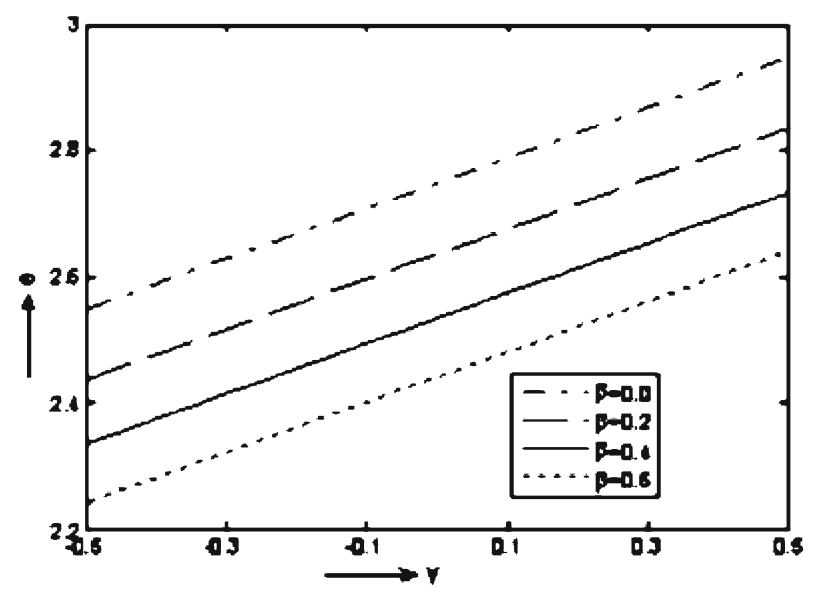

Fig. 19 Variation of temperature with y for different values of $\beta$ : $a=0.25, b=0.4, d=0.95, x=0.5$

narrow part of the channel $x \varepsilon[0.1,0.4]$ a much pressure gradient is required to maintain the same flux to pass it especially near $x=0.24$. From Fig. 12, it may be noted that $\beta$ decreases the maximum amplitude of $\frac{\mathrm{d} p}{\mathrm{~d} x}$. But the amplitude of pressure gradient is increased by increasing $F_{r}$ as given in Fig. 13. In Fig. 14 we have plotted $\frac{\mathrm{d} p}{\mathrm{~d} x}$ against $x$ to see the effect of changing inclination angle $\alpha$ and noticed that as inclined angle increases, the amplitude of pressure gradient increases for different values $x$.

The variations of pressure rise $\Delta p_{\lambda}$ per wave length against the mean flow rate $\Theta$ of an asymmetric inclined channel are illustrated in Figs. 15, 16 and 17 for various physical parameters. In these figures specific attention is given to the pumping regions, peristaltic pumping $\left(\Theta>0, \Delta p_{\lambda}>0\right)$, augmented pumping $(\Theta>0, \Delta P<0)$ and retrograde $\left(\Theta<0, \Delta p_{\lambda}>0\right)$. The effects of $\alpha$ on $\Delta p_{\lambda}$ are shown in Fig. 15. It shows that there is a linear relation between $\Delta p_{\lambda}$ and $\Theta$ and an increase of $\alpha$ results in increase in pumping rate. Figure 16 reveals that $\Delta p_{\lambda}$ decreases with increasing $F_{r}$ and is uniform for all pumping region.These observations show good agreement with the results of Nadeem and Akbar [30]. Figure 17 shows that the retrograde pumping rate decreases with increase of $\beta$ and decreases as flow rate increases. However, opposite effects are noticed for the case of augmented pumping.

To explicitly see the effects of various parameters,say $\gamma$ and $\beta$ on temperature, Eq. (43) has been numerically evaluated and the results are presented in Figs. 18, 19. We observed from Fig. 18 that the temperature increases with increase of $\gamma$ at the lower portion of the channel but effect is reverse at the upper portion of the channel. Further, it can be noted that the temperature at the lower wall is minimum and it increases slowly towards the upper wall. Also it is observed from Fig. 19 that there is a linear relation between $\theta$ and $y$ and temperature decreases with increasing $\beta$. 


\section{Conclusions}

In this work, the effect of slip conditions on peristaltic flow of Johnson-Segalman fluid in an asymmetric inclined channel are studied. The closed-form analytical solutions of the problem under long wavelength approximations are obtained. The results are discussed through graphs and concluded the following observations:

(1) the size of the trapped bolus increases by increasing $\Theta$ and $W_{e}$ but effect is reverse for $\beta$.

(2) The magnitude of axial velocity increases with the increase in $\phi$ and decreases by increasing $\beta$ near the wall.

(3) The amplitude of pressure gradient increases by increasing $F_{r}$ and $\alpha$ but effect is opposite for $\beta$.

(4) The peristaltic pumping rate increases with increase of $\alpha$ and decreases by increasing $F_{r}$ and is uniform for all pumping region. The retrograde pumping rate decreases with increase of $\beta$ but effect is reverse for augmented pumping.

(5) The temperature field decreases with the increase in $\beta$ while with the increase in $\gamma$ the temperature field increases.

These observations agree quantitatively well with those of existing results as mentioned above and hence the applicability of the present model is well validated. It is hoped that the results obtained will not only provide useful information for applications in various field of medical science but also serve as a complement to the previous studies.

Acknowledgments The author wishes to express his sincere thanks to the honorable referees for their valuable comments and suggestions to improve the quality of the paper.

Open Access This article is distributed under the terms of the Creative Commons Attribution License which permits any use, distribution, and reproduction in any medium, provided the original author(s) and the source are credited.

\section{References}

1. Ali, N.; Wang, Y.; Hayat, T.; Oberlack, M.: Long wavelength approximation to peristaltic flow of an Oldroyd 4-constant fluid in a planar channel. Biorheology 45, 611-628 (2008)

2. Das, K.: Heat transfer on peristaltic transport wiyh slip condition in an asymmetric porous channel. IJE Trans. B Appl. 24(3), 293-301 (2011)

3. Das, K.: MHD peristaltic pumping of a Johnson-Segalman fluid in an inclined asymmetric porous channel. Indian J. Math. AMS 53(2) (2011, accepted)

4. Derek, C.; Tretheway, D.C.; Meinhart, C.D.: Apparent fluid slip at hydrophobic microchannel walls. Phys. Fields. 14, L9-L12 (2002)

5. Elshahed, M.; Haroun, M.: Peristaltic flow of a Johnson-Segalman fluid under effect of a magnetic field. Math. Probl. Eng. 6, 663-677 (2005)

6. Fung, Y.C.; Yih, C.S.: Peristaltic transport. Trans. ASME J. Appl. Mech. 33, 669-675 (1968)

7. Haroun, M.H.: Effect of Deborah number and phase difference peristaltic transport of a third order fluid in an asymmetric channel. Commun. Nonlinear Sci. Numer. Simulat. 12, 1464-1480 (2007)

8. Haroun, M.H.: Non-linear peristaltic flow of a fourth grade fluid in an inclined asymmetric channel. Comput. Mater. Sci. 39, 324-333 (2007)

9. Hayat, T.; Wang, Y.; Siddiqui, A.M.; Asghar, K.: Peristaltic transport of a third order fluid in a circular tube. Math. Models Methods Appl. Sci. 12, 1691-1706 (2002)

10. Hayat, T.; Wang, Y.; Siddiqui, A.; Hutter, K.: Peristaltic flow of a Johnson-Segalman fluid in a planner channel. Math. Probl. Eng. 1, 1-23 (2003)

11. Hayat, T.; Ali, N.: Peristaltically induced motion of MHD third grade fluid in a deformable tube. Phys. A. 370, 225-239 (2006)

12. Hayat, T.; Hina, S.; Ali, N.: Simultaneous effects of slip and heat transfer on the peristaltic flow. Commun. Nonlinear Sci. Numer. Simul. 15, 1526-1537 (2010)

13. Hayat, T.; Mehmood, O.U.: Slip effects on MHD flow of third order fluid in a planar channel. Commun. Nonlinear Sci. Numer. Simul. 16, 1363-1377 (2011)

14. Johnson, M.W. Jr.; Segalman, D.A: Model for viscoelastic fluid behavior which allows non-affine deformation. J. Non Newton. Fluid Mech. 2, 255-270 (1977)

15. Kolkka, R.W.; Malkus, D.S.; Hansen, M.G.; Ierley, G.R.: Spurt phenomena of the Johnson-Segalman fluid and related models. J. Non Newton. Fluid Mech. 29, 303-335 (1988)

16. Kothandapani, M.; Srinivas, S.: Peristaltic transport of a Jeffrey fluid under the effect of magnetic field in an asymmetric channel. Int. J. Nonlinear Mech. 43, 915-924 (2008)

17. Latham, T.W.: Fluid motion in peristaltic pump, S.M. Thesis, MIT (1966)

18. Malkus, D.S.; Nohel, J.A.; Plohr, B.J.: Dynamics of shear flow of a non-Newtonian fluid. J. Comput. Phys. 87, 464-487 (1990) 
19. McLeish, T.C.B.; Ball, R.C.: A molecular approach to the spurt effect in polymer melt flow. J. Polymer Sci. Part B. 24, 1735-1745 (1986)

20. Mekheimer, S. Kh.: Peristaltic flow of blood under the effect of magnetic field in a non uniform channel. Appl. Math. Comput. 153, 763-777 (2004)

21. Mekheimer, S. Kh.: Effect of the induced magnetic field on peristaltic flow of a couple stress fluid. Phys. Lett. A. 372, 4271-4278 (2008)

22. Mekheimer, S. Kh.; Abd elmaboud, Y.: Peristaltic flow of a couple stress fluid in an annulus: application of an endoscope. Phys. A. 387, 2403-2415 (2008)

23. Mishra, M.; Rao, A.R.: Peristaltic transport of a Newtonian fluid in an asymmetric channel. Zeitschrift für angewandte Mathematik und Physik 54, 440-532 (2004)

24. Nadeem, S.; Akbar, N.S.: Numerical solutions of peristaltic flow of a Newtonian fluid under the effects of magnetic field and heat transfer in a porous concentric tubes. Zeitschrift fur Naturforschung 65a, 1-12 (2010)

25. Nadeem, S.; Akbar, N.S.: Effects of temperature dependent viscosity on peristaltic flow of a Jeffrey-six constant fluid in a non-uniform vertical tube. Commun. Nonlinear Sci. Numer. Simul. 15, 3950-3964 (2010)

26. Nadeem, S.; Akbar, N.S.: Influence of heat and mass transfer on a peristaltic motion of a Jeffrey-six constant fluid in an annulus. Heat Mass Transf. 46, 485-493 (2010)

27. Nadeem, S.; Akram, S.: Slip effects on the peristaltic flow of a Jeffrey fluid in an asymmetric channel under the effect of induced magnetic field. Int. J. Numer. Methods Fluids 63, 374-394 (2010)

28. Nadeem, S.; Akbar, N.S.: Influence of heat and chemical reactions on the peristaltic flow of a Johnson Segalman fluid in a vertical asymmetric channel with induced MHD. Taiwan Inst. Chem. Eng. 42, 58-66 (2011)

29. Nadeem, S.; Akram, S.: Heat transfer in a peristaltic flow of MHD fluid with partial slip. Commun. Nonlinear Sci. Numer. Simulat. 15, 312-321 (2010)

30. Nadeem, S.; Akbar, N.S.: Influence of heat transfer on peristaltic transport of a Johnson-Segalman fluid in an inclined asymmetric channel. Commun. Nonlinear Sci. Numer. Simulat. 15, 2860-2877 (2010)

31. Nadeem, S.; Akbar, N.S.; Bibi, N.; Ashiq, S.: Influence of heat and mass transfer on peristaltic flow of a third order fluid in a Diverging tube. Commun. Nonlinear Sci. Numer. Simul. 15, 2916-2931 (2010)

32. Shapiro, A.H.; Jaffrin, M.Y.; Weinberg, S.L.: Peristaltic pumping with long wavelength at low Reynolds number. J. Fluid Mech. 37, 799-825 (1969)

33. Srinivas, S.; Pushparaj, V.: Non-linear peristaltic transport in an inclined asymmetric channel. Commun. Nonlinear Sci. Numer. Simul. 13, 1782-1795 (2008)

34. Wang, Y.; Hayat, T.; Hutter, K.: Peristaltic flow of a Johnson-Segalman fluid through a deformable tube. Theor. Comput. Fluid Dyn. 21, 369-380 (2007) 